\title{
EPR Spectroscopy Study of the Radical Cation Cluster $\left(\eta^{5}-\mathrm{C}_{5} \mathrm{H}_{5}\right)(\mathrm{CO})_{5} \operatorname{ReFePt}\left(\mu_{3}-\mathrm{C}=\mathrm{CHPh}\right)\left(\eta^{2}-\mathrm{Ph}_{2} \mathbf{P}\left(\mathrm{CH}_{2}\right)_{2} \mathbf{P P h}_{2}\right)$
}

\author{
Nikolay G. Maksimova, Victor V. Verpekin', \\ Dmitry V. Zimonin*a, Galina V. Burmakina ${ }^{\mathrm{a}, \mathrm{b}}$, \\ Oleg S. Chudin a and Anatoly I. Rubaylo ${ }^{\mathrm{a}, \mathrm{b}}$ \\ anstitute of Chemistry and Chemical Technology SB RAS \\ FRC "Krasnoyarsk Science Center SB RAS" \\ 50/24 Akademgorodok, Krasnoyarsk, 660036, Russia \\ ${ }^{b}$ Siberian Federal University \\ 79 Svobodny, Krasnoyarsk, 660041, Russia
}

Received 17.10.2019, received in revised form 02.11.2019, accepted 21.11.2019

The chemical oxidation of the cluster $C p \boldsymbol{R e F e P t}\left(\mu_{3}-C=C H P h\right)(C O)_{5}(d p p e)\left(C p=\eta^{5}-C_{5} H_{5}\right.$, dppe $=\eta^{2}-$ $\left.\mathrm{Ph}_{2} \mathrm{P}\left(\mathrm{CH}_{2}\right)_{2} \mathrm{PPh}_{2}\right)$ resulted in a radical cation $\left[\mathrm{Cp} \boldsymbol{R e F e P t}\left(\mu_{3}-\mathrm{C}=\mathrm{CHPh}\right)(\mathrm{CO})_{5}(\text { dppe })\right]^{+*}$ that is sufficiently stable only at low temperature. An electronic structure of the radical cation was studied by EPR and following parameters were obtained by comparison of the experimental and model spectrum: $g_{x}=2.070 g_{y}=2.0295 g_{z}=1.997 ; A_{x}\left({ }^{\beta l} P\right)=17 A_{y}\left({ }^{\beta l} P\right)=49 A_{z}\left({ }^{1 l} P\right)=35(G s) ; A_{x}\left({ }^{195} P t\right)=62 A_{y}\left({ }^{195} P t\right)=45$ $A_{z}\left({ }^{195} P t\right)=105(G s)$. An unpaired electron is seen to be mainly concentrated on the iron atom (85-90\%) and partially on the platinum atom (10-15\%). Further transformation of the radical cation led to the formation of the binuclear complex $\mathrm{Cp}(\mathrm{CO})_{2} \operatorname{RePt}(\mu-\mathrm{C}=\mathrm{CHPh})($ dppe) and the Fe-carbonyl fragment.

Keywords: EPR spectroscopy, radical cation, redox transformations, trinuclear clusters, vinylidene ligands, rhenium, iron, platinum, diphosphine ligand.

Citation: Maksimov N.G., Verpekin V.V., Zimonin D.V., Burmakina G.V., Chudin O.S., Rubaylo A.I. EPR spectroscopy study of the radical cation cluster $\left(\eta^{5}-\mathrm{C}_{5} \mathrm{H}_{5}\right)(\mathrm{CO})_{5} \operatorname{ReFePt}\left(\mu_{3}-\mathrm{C}=\mathrm{CHPh}\right)\left(\eta^{2}-\mathrm{Ph}_{2} \mathrm{P}_{(}\left(\mathrm{CH}_{2}\right)_{2} \mathrm{PPh}_{2}\right)$, J. Sib. Fed. Univ. Chem., 2019, 12(4), 573-579. DOI: 10.17516/1998-2836-0152.

(C) Siberian Federal University. All rights reserved

* Corresponding author E-mail address: zimonind89@mail.ru 


\title{
Изучение методом ЭПР-спектроскопии
}

\section{катион-радикала кластера \\ $\left(\eta^{5}-C_{5} H_{5}\right)(C O)_{5} \operatorname{ReFePt}\left(\mu_{3}-\mathrm{C}=\mathrm{CHPh}\right)\left(\eta^{2}-\mathrm{Ph}_{2} \mathbf{P}\left(\mathrm{CH}_{2}\right)_{2} \mathrm{PPh}_{2}\right)$}

\author{
Н.Г. Максимов ${ }^{\mathrm{a}}$, В.В. Верпекин ${ }^{\mathrm{a}}$, Д.В. Зимонин ${ }^{\mathrm{a}}$, \\ Г.В. Бурмакина ${ }^{\mathrm{a}, \boldsymbol{\sigma}}$, О.С. Чудин ${ }^{\mathrm{a}}$, А.И. Рубайло ${ }^{\mathrm{a}, \bar{\sigma}}$ \\ ${ }^{a}$ Институт химии и химической технологии СО РАН \\ ФИЦ «Красноярский научный иентр СО РАН» \\ Россия, 660036, Красноярск, Академгородок, 50/24 \\ ${ }^{6}$ Сибирский федеральный университет \\ Россия, 660041, Красноярск, пр. Свободный, 79
}

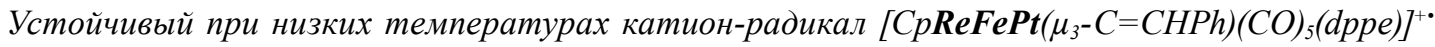
$\left(C p=\eta^{5}-C_{5} H_{5}\right.$, dppe $\left.=\eta^{2}-P h_{2} P\left(C_{2}\right)_{2} P P h_{2}\right)$, полученный химическим окислением кластера CpReFePt $\left(\mu_{3}-\mathrm{C}=\mathrm{CHPh}\right)(\mathrm{CO})_{5}($ dppe), изучен методом ЭПР-спектроскопии. На основании сопоставления экспериментальных и модельных ЭПР-спектров катион-радикала кластера установлены его характеристики: $g_{x}=2.070 g_{y}=2.0295 g_{z}=1.997 ; A_{x}\left({ }^{\beta} P\right)=17 A_{y}\left({ }^{1} P\right)=49$ $A_{z}\left({ }^{11} \mathrm{P}\right)=35(\mathrm{Gs}) ; A_{x}\left({ }^{95} \mathrm{Pt}\right)=62 A_{y}\left({ }^{95} \mathrm{Pt}\right)=45 A_{z}\left({ }^{195} \mathrm{Pt}\right)=105(\mathrm{Gs})$. Показано, что в образуюшемся парамагнитном соединении спиновая плотность сосредоточена на атоме железа (85-90\%) $u$ частично на атоме платины (10-15\%). Дальнейшее превращение катион-радикала протекает c образованием биядерного комплекса $\mathrm{Cp}(\mathrm{CO})_{2} \boldsymbol{\operatorname { R e P t }}(\mu-\mathrm{C}=\mathrm{CHPh})(\mathrm{dppe)}$ и $\mathrm{Fe}$-карбонильного фрагмента.

Ключевые слова: ЭПР-спектроскопия, катион-радикал, редокс-превращения, кластеры, винилиденовые лиганды, рений, железо, платина, дифосфиновый лиганд.

\section{Введение}

Полиметаллические кластерные соединения, содержащие различные ненасыщенные углеводородные лиганды, привлекают исследователей возможностями их применения в медицине и катализе, в разработке новых материалов и молекулярных машин [1-3]. В связи с этим много внимания уделяется изучению химических и физико-химических свойств кластерных соединений [4-6]. Важным направлением в химии гетерометаллических кластеров является изучение их редокс-превращений [7-9]. Для проведения таких исследований необходимо совместное использование электрохимических и спектроскопических методов. Так, например, нам удалось изучить пути редокс-превращений кластеров $\mathrm{Cp} \operatorname{ReFePd}\left(\mu_{3}-\mathrm{C}=\mathrm{CHPh}\right)(\mathrm{CO})_{5}(\mathrm{P}-\mathrm{P})$ [P-P $=\mathrm{Ph}_{2} \mathrm{P}\left(\mathrm{CH}_{2}\right)_{\mathrm{n}} \mathrm{PPh}_{2}, \mathrm{n}=2$ (dppe), 3 (dppp)], выявить и охарактеризовать их относительно устойчивые катион-радикалы $[10,11]$.

Ранее нами были синтезированы трехъядерные кластеры $\mathrm{CpReFePt}\left(\mu_{3}-\mathrm{C}=\mathrm{CHPh}\right)(\mathrm{CO})_{5}(\mathrm{P}-\mathrm{P})$ $\left(\mathrm{P}-\mathrm{P}=\mathrm{Ph}_{2} \mathrm{P}\left(\mathrm{CH}_{2}\right)_{\mathrm{n}} \mathrm{PPh}_{2}, \mathrm{n}=2\right.$ (dppe), 3 (dppp)) [12]. Изучены их электрохимические свойства и установлено, что окисление $\operatorname{ReFePt}$ кластеров приводит к образованию биядерных комплек- 
сов $\mathrm{Cp}(\mathrm{CO})_{2} \operatorname{Re}(\mu-\mathrm{C}=\mathrm{CHPh}) \mathbf{P t}(\mathrm{P}-\mathrm{P})(\mathrm{P}-\mathrm{P}=\mathrm{dppe}, \mathrm{dppp})$ и фрагмента $\left[\mathbf{F e}(\mathrm{CO})_{3}\right]^{+*}[11]$. Однако существование промежуточных катион-радикалов этих ReFePt кластеров доказано не было. Поэтому настоящая работа посвящена получению и изучению продуктов окисления кластера $\mathrm{Cp} \operatorname{ReFePt}\left(\mu_{3}-\mathrm{C}=\mathrm{CHPh}\right)(\mathrm{CO})_{5}(\mathrm{dppe})(\mathbf{1})$ методом ЭПР-спектроскопии.

\section{Экспериментальная часть}

Все операции проводили в атмосфере аргона или азота. В качестве растворителя использовали свежеперегнанный дихлорметан, хранящийся в атмосфере аргона и предварительно очищенный от примесей, следов воды и кислорода путем перегонки над соответствующими осушителями [13]. Кластер $\mathrm{Cp \operatorname {ReFePt }}\left(\mu_{3}-\mathrm{C}=\mathrm{CHPh}\right)(\mathrm{CO})_{5}(\mathrm{dppe})$ (1) и катион-радикал ферроцения тетрафторбората $\left[\mathrm{Cp}_{2} \mathrm{Fe}\right]\left[\mathrm{BF}_{4}\right]$ синтезировали по известным методикам [12, 14].

Катион-радикал $\mathbf{1}^{+\bullet}$ получали по реакции кластера $\mathrm{CpReFePt}\left(\mu_{3}-\mathrm{C}=\mathrm{CHPh}\right)(\mathrm{CO})_{5}(\mathrm{dppe})(\mathbf{1}) \mathrm{c}$ ферроцением тетрафторборатом. К 2 мл 1 мМ раствора кластера 1 добавляли 2 мл 1 мМ раствора $\left[\mathrm{Cp}_{2} \mathrm{Fe}\right]\left[\mathrm{BF}_{4}\right]$ при $233 \mathrm{~K}$, цвет реакционной смеси менялся с красно-розового на краснооранжевый. После приготовления один образец сразу помещали в кварцевую ампулу и замораживали в жидком азоте, другой оставляли в течение 5 мин при $233 \mathrm{~K}$, затем переносили в ампулу и замораживали. Опыты проводили в трех параллелях. Спектры ЭПР регистрировались в режиме CW на приборе ELEXYSE-580 (Bruker ФРГ) при температуре 86-88 К в кварцевых ампулах. Моделирование спектров ЭПР проводили с использованием программы XSophe (Bruker ФРГ).

\section{Результаты и обсуждение}

Электрохимическое исследование кластера $\mathrm{Cp} \operatorname{ReFePt}\left(\mu_{3}-\mathrm{C}=\mathrm{CHPh}\right)(\mathrm{CO})_{5}(\mathrm{dppe})(\mathbf{1})$ показало [11], что его окисление протекает в несколько стадий и приводит на первой одноэлектронной стадии к образованию катион-радикала $\mathbf{1}^{+\cdot}$. Значение потенциала этой стадии указывает на возможность использования ферроцения тетрафторбората $\left[\mathrm{Cp}_{2} \mathrm{Fe}_{2}\right]\left[\mathrm{BF}_{4}\right][14]$ для осуществления одноэлектронного окисления $\mathbf{1}$ химическим путем. Поэтому для доказательства того, что окисление ReFePt кластера 1 протекает через стадию образования относительно устойчивого катион-радикала $\mathbf{1}^{+}$, были проведены эксперименты по химическому окислению его растворов с использованием $\left[\mathrm{Cp}_{2} \mathrm{Fe}\right]\left[\mathrm{BF}_{4}\right]$ при минус $40{ }^{\circ} \mathrm{C}$ с последующей заморозкой полученных образцов и их дальнейшем исследованием методом ЭПР-спектроскопии (см. экспериментальную часть).

В спектрах ЭПР полученных образцов (рис. 1) наблюдается появление характерных сигналов от парамагнитных центров $(\mathrm{S}=1 / 2)$ с ромбической анизотропией g-фактора. Присутствие в спектрах ЭПР дополнительных переходов можно связать с наличием сверхтонкой структуры (CTC) от ядер, имеющих ненулевые магнитные моменты. Количество дополнительных переходов указывает на наличие двух ядерных спинов ( $\mathrm{I}=0$ и $\mathrm{I}=1 / 2)$, что свидетельствует о присутствии в растворе изотопов. Сопоставление этих факторов с составом кластера 1 указывает на то, что сверхтонкое расщепление протекает на ядрах ${ }^{195} \mathrm{Pt}(\mathrm{I}=1 / 2$ и $33 \%$ от общего содержания ядер платины). Для определения наличия СТС от ядер платины и более точного нахождения параметров ЭПР наблюдаемых парамагнитных центров было проведено моделирование спек- 


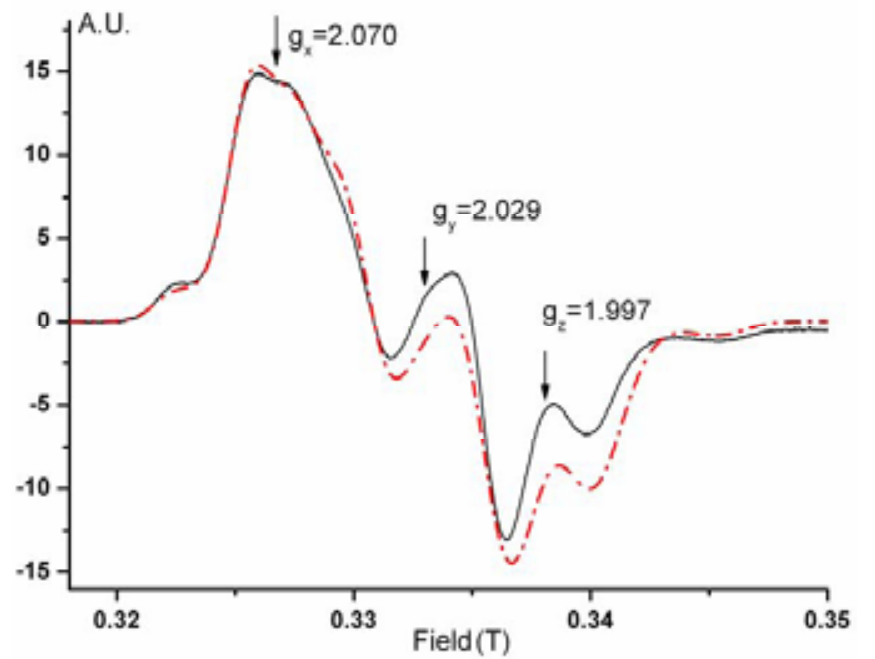

Рис. 1. Спектры ЭПР (T = $90 \mathrm{~K})$ замороженной реакционной смеси $\mathrm{CpReFePt}\left(\mu_{3}-\mathrm{C}=\mathrm{CHPh}\right)(\mathrm{CO})_{5}(\mathrm{dppe})$ (1) $(1 \mathrm{MM})$ и $\left[\mathrm{Cp}_{2} \mathrm{Fe}\right]\left[\mathrm{BF}_{4}\right](1 \mathrm{MM})$ в $\mathrm{CH}_{2} \mathrm{Cl}_{2}$ (сплошная линия) и модельного спектра катион-радикала $\mathbf{1}^{+*}$ (штрихпунктирная линия)

Fig. 1. EPR spectrum $(\mathrm{T}=90 \mathrm{~K})$ of the frozen reaction mixture of $\mathrm{Cp} \operatorname{ReFePt}\left(\mu_{3}-\mathrm{C}=\mathrm{CHPh}\right)(\mathrm{CO})_{5}(\mathrm{dppe})(\mathbf{1})(1$ $\mathrm{mM})$ and $\left[\mathrm{Cp}_{2} \mathrm{Fe}\right]\left[\mathrm{BF}_{4}\right](1 \mathrm{mM})$ in $\mathrm{CH}_{2} \mathrm{Cl}_{2}$ (the solid line) and the simulated EPR spectrum of the $\mathbf{1}^{+*}$ radical cation (the dash-dot line)

тров. На рис. 1 представлен спектр ЭПР образца окисленного ReFePt кластера 1 и модельного спектра. Параметры ЭПР, полученные на основании сопоставления экспериментальных и модельных спектров, следующие:

$$
\begin{aligned}
& \mathrm{g}_{\mathrm{x}}=2.070 \mathrm{~g}_{\mathrm{y}}=2.0295 \mathrm{~g}_{\mathrm{z}}=1.997 ; \mathrm{A}_{\mathrm{x}}\left({ }^{31} \mathrm{P}\right)=17 \mathrm{~A}_{\mathrm{y}}\left({ }^{31} \mathrm{P}\right)=49 \mathrm{~A}_{\mathrm{z}}\left({ }^{31} \mathrm{P}\right)=35(\mathrm{Gs}) \\
& \mathrm{A}_{\mathrm{x}}\left({ }^{195} \mathrm{Pt}\right)=62 \mathrm{~A}_{\mathrm{y}}\left({ }^{195} \mathrm{Pt}\right)=45 \mathrm{~A}_{\mathrm{z}}\left({ }^{195} \mathrm{Pt}\right)=105(\mathrm{Gs}) .
\end{aligned}
$$

Полученные величины значений констант СТС от ядер ${ }^{195} \mathrm{Pt}$ для катион-радикала $\mathbf{1}^{+\bullet}$ в 6-8 раз меньше литературных данных для платиносодержащих комплексов [15], что позволяет оценить величины спиновой плотности неспаренного электрона, локализованного на атоме платины, в 10-15\%. Основная часть спиновой плотности (85-90\%) сосредоточена на атоме железа, имеющего нулевой магнитный момент $(\mathrm{I}=0)$ и не влияющего на СТC.

В исходном кластере 1 лиганд 1,2-бис(дифенилфосфино)этан (dppe) хелатно координируется с атомом платины [12]. Однако наблюдаемая CTC $(\mathrm{I}=1 / 2)$, отнесенная $\mathrm{K}^{31} \mathrm{P}$, соответствует только одному атому фосфора и $\eta^{1}$-координации лиганда dppe, следовательно, связь с неспаренным электроном, локализованным на платине, образует только один атом фосфора, что приводит к частичному переносу неспаренного электрона на этот атом фосфора.

На основании полученных результатов и информации о структуре кластера 1 строение катион-радикала $\mathbf{1}^{+\bullet}$ можно представить в виде двух резонансных форм $\mathbf{A}$ и $\mathbf{B}$ (рис. $2 a$ и $б$ ), в которых возможность локализации неспаренного электрона реализуется для атома как железа, так и платины.

Рассмотрение двух резонансных форм катион-радикала $\mathbf{1}^{+\cdot}$ показывает, что структура $\mathbf{A}$ маловероятна на основании данных ЭПР, так как для данной структуры можно ожидать пере- 


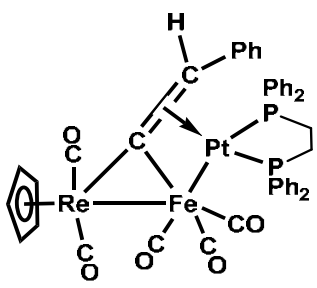

a

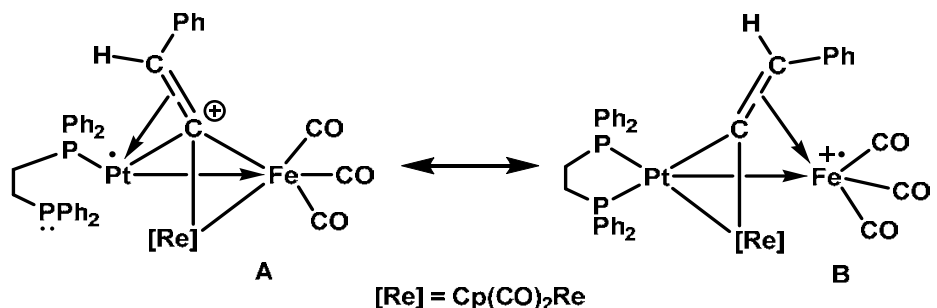

б

Рис. 2: a - Cтроение кластера $\mathrm{Cp} \operatorname{ReFePt}\left(\mu_{3}-\mathrm{C}=\mathrm{CHPh}\right)(\mathrm{CO})_{5}(\mathrm{dppe})(\mathbf{1}) ;$ б - строение возможных резонансных форм катион-радикала $\mathbf{1}^{+\cdot}$

Fig. 2: a - The structure of the cluster $\mathrm{CpReFePt}\left(\mu_{3}-\mathrm{C}=\mathrm{CHPh}\right)(\mathrm{CO})_{5}(\mathrm{dppe})(\mathbf{1}) ; \sigma$ - proposed resonance forms of the $\mathbf{1}^{+\bullet}$ radical cation

носа спиновой плотности с атома железа на атом рения. Это противоречит экспериментальным результатам, что связь $\mathrm{Re}-\mathrm{M}(\mathrm{M}=\mathrm{Pt}$ или $\mathrm{Fe})$ осуществляется только для того атома, на котором не локализована спиновая плотность, иначе в спектре ЭПР наблюдалась дополнительная СТС от атома рения. Поэтому более вероятно, что для катион-радикала предпочтительна форма $\mathbf{B}$, для которой характерно наличие координации двумя атомами фосфора. Это означает, что координационное состояние двух атомов фосфора формы В является неэквивалентным. То есть один из двух атомов фосфора взаимодействует с d-орбиталью платины, на которой локализирован неспаренный электрон, а другой атом, соответственно, не взаимодействует с этой орбиталью.

При выдержке во времени растворов смеси кластера 1 c $\left[\mathrm{Cp}_{2} \mathrm{Fe}\right]\left[\mathrm{BF}_{4}\right]$ (образец 2) происходит уменьшение интенсивности сигналов в спектре ЭПР без заметных изменений форм линий этих сигналов, это свидетельствует о дальнейшем превращении катион-радикала в диамагнитное соединение. После разморозки обоих образцов и выдерживании их при комнатной температуре в течение 30 сек катион-радикальные формы $\mathbf{1}^{+*}$ не обнаружены. Идентификация полученных продуктов методом ИК-спектроскопии показала, что в растворах образуется соответствующий биядерный $\boldsymbol{R e P t}$ комплекс.

Таким образом, на основании электрохимических и ЭПР спектроскопических данных доказано ранее выдвинутое предположение, что окисление ReFePt кластера 1 протекает через стадию образования относительно устойчивого катион-радикала $\mathbf{1}^{+*}$, дальнейшие превращения которого приводят к биядерному комплексу $\mathrm{Cp}(\mathrm{CO})_{2} \mathbf{R e}(\mu-\mathrm{C}=\mathrm{CHPh}) \mathbf{P t}(\mathrm{dppe})$ и фрагменту $\left[\mathrm{Fe}(\mathrm{CO})_{3}\right]^{+*}:$

$$
\begin{aligned}
& \mathrm{CpReFePt}\left(\mu_{3}-\mathrm{C}=\mathrm{CHPh}\right)(\mathrm{CO})_{5}(\mathrm{P}-\mathrm{P}) \stackrel{-\mathrm{e}}{\rightarrow}\left[\mathrm{CpReFePt}\left(\mu_{3}-\mathrm{C}=\mathrm{CHPh}\right)(\mathrm{CO})_{5}(\mathrm{P}-\mathrm{P})\right]^{+\bullet} \\
& {\left[\mathrm{CpReFePt}\left(\mu_{3}-\mathrm{C}=\mathrm{CHPh}\right)(\mathrm{CO})_{5}(\mathrm{P}-\mathrm{P})\right]^{+\cdot} \rightarrow \mathrm{Cp}(\mathrm{CO})_{2} \operatorname{RePt}(\mu-\mathrm{C}=\mathrm{CHPh})(\mathrm{P}-\mathrm{P})+\left[\mathrm{Fe}(\mathrm{CO})_{3}\right]^{+\cdot}}
\end{aligned}
$$

\section{Благодарность}

Работа выполнена в рамках государственного задания Института химии и химической технологии СО РАН (проект АААА-А17-117021310221-7) с использованием оборудования Красноярского регионального центра коллективного пользования ФИЦ КНЦ СО РАН 


\section{Список литературы}

1. Gervasio G., Sappa E., Secco A. Advances in the chemistry of alkyne-substituted homo- and heterometallic carbonyl clusters of the iron, cobalt and nickel triads. An update. J. Organomet. Chem. 2014. Vol. 751, P. 111-152.

2. Akita M., Koike T. Chemistry of polycarbon species: from clusters to molecular devices. Dalt. Trans. 2008. Vol. 27, P. 3523-3530.

3. Sánchez-Cabrera G., Zuno-Cruz F.J., Rosales-Hoz M.J. Reactivity of Alkyne-Substituted Transition Metal Clusters: An Overview. J. Clust. Sci. 2014. Vol 25(1), P. 51-82.

4. Buchwalter P., Rosé J., Braunstein P. Multimetallic catalysis based on heterometallic complexes and clusters. Chem. Rev. 2015. Vol. 115, P. 28-126.

5. Sculfort S., Braunstein P. Intramolecukar $\mathrm{d}^{10}-\mathrm{d}^{40}$ interactions in heterometallic clusters of the transition metals. Chem. Soc. Rev. 2011. Vol. 40(5), P. 2741-2760.

6. Puddephatt R.J. Compounds with platinum-metal bonds: A personal account. J. Organomet. Chem. 2017. Vol. 849-850, P. 268-278.

7. Lemoine P. Progress in cluster electrochemistry. Coord. Chem. Rev. 1988. Vol. 83, P. 169197.

8. Geiger W.E. Organometallic electrochemistry: origins, development, and future. Organometallics 2007. Vol. 26, P. 5738-5765

9. Drake S.R. Electrochemical studies on iron, ruthenium and osmium carbonyl clusters. Polyhedron 1990. Vol. 9(4), P. 455-474.

10. Burmakina G.V., Verpekin V.V., Maksimov N.G., Zimonin D.V., Piryazev D.A., Chudin O.S., Rubaylo A.I. Electrochemically induced trasmormation of bi- and trinuclear heterometallic vinylidene complexes containing Re, Pd and Fe. Inorg. Chim. Acta 2017. Vol. 463, P. 70-79.

11. Бурмакина Г.В., Верпекин В.В., Зимонин Д.В., Чудин О.С., Ергаев Р.О., Рубайло А.И. Электрохимическое изучение фенилвинилиденовых кластеров с остовом ReFePt, содержащих хелатные дифосфиновые лиганды. Журн. Сибирского федерального университета. Химия. 2017. T. 10(3), C. 358-367. [Burmakina G.V.,Verpekin V.V., Zimonin D.V., Chudin O.S., Ergaev R.O., Rubaylo A.I. Electrochemical study of phenylvinylidene clusters containing ReFePt metal cores and chelate diphosphine ligands. Journal of Siberian Federal University. Chemistry. 2017. Vol. 10(3), P. 358-367. (In Russ.)]

12. Верпекин В.В., Кондрасенко А.А., Ергаев Р.О., Чудин О.С., Павленко Н.И., Рубайло А.И. Фенилвинилиденовые кластеры с остовом ReFePt, содержащие хелатные дифосфиновые лиганды при атоме платины. Журн. Сибирского федерального университета. Химия. 2017. T. 10(2), C. 239-249. [Verpekin V.V., Kondrasenko A.A., Ergaev R.O., Chudin O.S., Pavlenko N.I., Rubaylo A.I. Phenylvinylidene clusters containing ReFePt metal cores and helate diphosphine ligands at the platinum atom. Journal of Siberian Federal University. Chemistry. 2017. Vol. 10(2), P. 239-249. (In Russ.)]

13. Вайсбергер Ф., Проскауэр Э., Диддик Дж., Тупс Э. Органические растворители. Физические свойства и методы очистки. М.: Иностр. лит., 1958. 519 с. [Weissberger F., Proskauer E., Diddick J., Tups E. Organic solvents. Physical properties and cleaning methods. M.; Inostr. lit., 1958. 519 p. (In Russ.)] 
14. Connelly N.G., Geiger W.E. Chemical redox agents for organometallic chemistry. Chem. Rev. 1996. Vol. 96, P. 877.

15. Alonso P.J., Alcalá R., Usón R., Forniés J. EPR study of mononuclear Pt(III) organometallic complexes. J. Phys. Chem. Sol. 1991. Vol. 52(8), P. 975-978. 